\title{
Is the error on first-principles volume predictions absolute or relative?
}

\author{
Kurt Lejaeghere,${ }^{1}$ Louis Vanduyfhuys, ${ }^{1}$ Toon Verstraelen, ${ }^{1}$ Veronique Van Speybroeck, ${ }^{1}$ and Stefaan Cottenier ${ }^{1,2}$ \\ ${ }^{1}$ Center for Molecular Modeling, Ghent University, \\ Technologiepark 903, BE-9052 Zwijnaarde, Belgium \\ ${ }^{2}$ Department of Materials Science and Engineering, \\ Ghent University, Technologiepark 903, BE-9052 Zwijnaarde, Belgium
}

\begin{abstract}
Many benchmarks of density-functional theory with respect to experiment suggest the error on predicted equilibrium volumes to scale with the volume. Relative volume errors are therefore often used as a decisive argument to select one exchange-correlation functional over another. We show that the error on the volume (after correcting for systematic deviations) is only approximately relative. A simple analytic model, validated by rigorous Monte Carlo simulations, reveals that a more accurate error estimate can be derived from the inverse of the bulk modulus. This insight is not only instrumental for the selection or design of suitable functionals. It also calls for a new attitude towards computational errors: to report computational errors on electronic-structure calculations, identify systematic deviations and distinguish between relative and absolute effects.
\end{abstract}

Keywords: density-functional theory, crystal volume, uncertainty quantification, equation of state

\section{INTRODUCTION}

Over the last few decades, powerful computers and advanced computational approaches have revolutionized materials science. Whereas first-principles methods were originally limited to a posteriori analyses of experimentally studied compounds, they now make it possible to investigate materials prior to experiment and at significantly lower costs. As a result, computational materials design has become a research discipline in its own right. However, a compromise between accuracy and available resources is inevitable. Applied methods vary from very approximate yet cheap (e.g. classical force fields ${ }^{1,2}$ ) to nearly exact yet terribly expensive (e.g. full configuration interaction ${ }^{3}$ ). A popular middle ground is densityfunctional theory $(\mathrm{DFT})^{4,5}$, which produces qualitatively acceptable predictions at a reasonable cost for many systems, provided a good choice is made for the so-called exchange-correlation functional. Nevertheless, the obtained results are still approximate, and the accuracy of the DFT calculations is determined by the selected functional. Understanding the expected deviations between DFT and experimental values is therefore essential to assess the reliability of a prediction and choose an appropriate functional correspondingly.

Several benchmark studies in the literature seek to evaluate the differences between DFT predictions and experimental results. Although these kind of studies are mainly limited to molecular systems ${ }^{7-11}$, a number of exhaustive solid-state tests are available as well. ${ }^{11-16}$ However, conclusions drawn in these works may be significantly affected by the choice of the error model. ${ }^{17}$ This is nicely illustrated by the equilibrium volume, or equivalently the lattice parameter. Most benchmarks list both absolute (mean error, mean absolute error) and relative differences with respect to experiment (mean relative error, mean absolute relative error) ${ }^{12-15}$ while other articles express the community intuition that errors should scale in a relative way (i.e. smaller errors on smaller volumes) and only mention the latter ${ }^{11,16}$. The volume of beryllium $\left(7.8 \AA^{3}\right.$ /atom experimentally), for example, is thus expected to be predicted more accurately with DFT than that of sodium $\left(37.2 \AA^{3}\right.$ /atom) or barium $\left(62.3 \AA^{3} /\right.$ atom $) .{ }^{6}$ In addition to these relatively simple approaches, some authors also assessed DFT errors more rigorously. Some of the present authors ${ }^{6,18}$, for example, applied a linear regression between experimental and DFT results to distinguish between systematic and residual deviations. In the current article, a systematic error denotes the predictable over- or underestimation of DFT compared to experiment, which can be corrected for by means of a regression analysis. The remaining unpredictable (yet deterministic) fluctuation is denoted as residual error. In References 6 and 18, we did not observe the residual errors on the volume to behave in a purely absolute or relative way (see Figure 1(a)). Pernot et al. ${ }^{19}$ applied a more general Bayesian Model Selection to determine the most appropriate polynomial degree to describe the systematic bias between predictions with several functionals and experiment. They also found a linear relationship to be most suitable, and expressed the remaining discrepancies in terms of method inadequacy and parametric uncertainty of the fit, using a virtual measurement framework. ${ }^{20}$ In this way, they found the prediction uncertainty on lattice parameters to increase as a function of the lattice parameter itself. Finally, Mortensen et al. ${ }^{21}$ extracted error estimates per element by varying the generalized-gradient approximation (GGA) functional itself according to a Gaussian distribution. This distribution was tuned to a least-squares fit of DFT-GGA cohesive energies to experimental results. Although the resulting error bars were found to scale nicely with the deviation from experiment, the study did not provide a definite answer on the nature of the volume error either.

It therefore remains unclear whether the error on $V_{0}$ should be an absolute or a relative one. The error on the volume is indeed larger for $\mathrm{Ba}$ than for $\mathrm{Be}$, for example, 
but not larger than for Na (Figure 1(a)). Nevertheless, insight in the error is critical for the comparison, ranking and construction of high-accuracy functionals. The present study aims to answer that question, based on the properties of a solid-state equation of state. After formulating our primary ansatz, we will approach the problem from both an analytic and a numerical (Monte Carlo) point of view. In each case, we will assume that systematic deviations (such as the $4 \%$ volume overestimation ${ }^{6}$

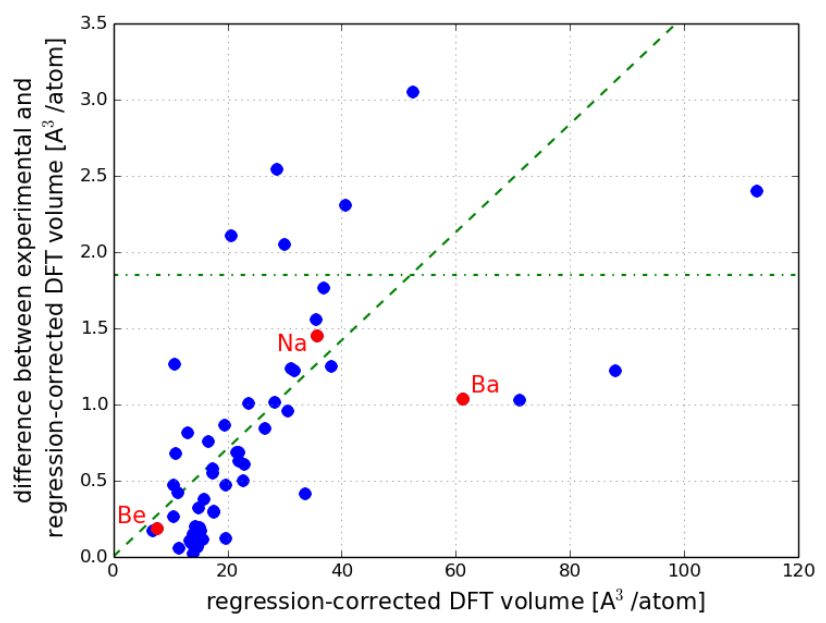

(a)

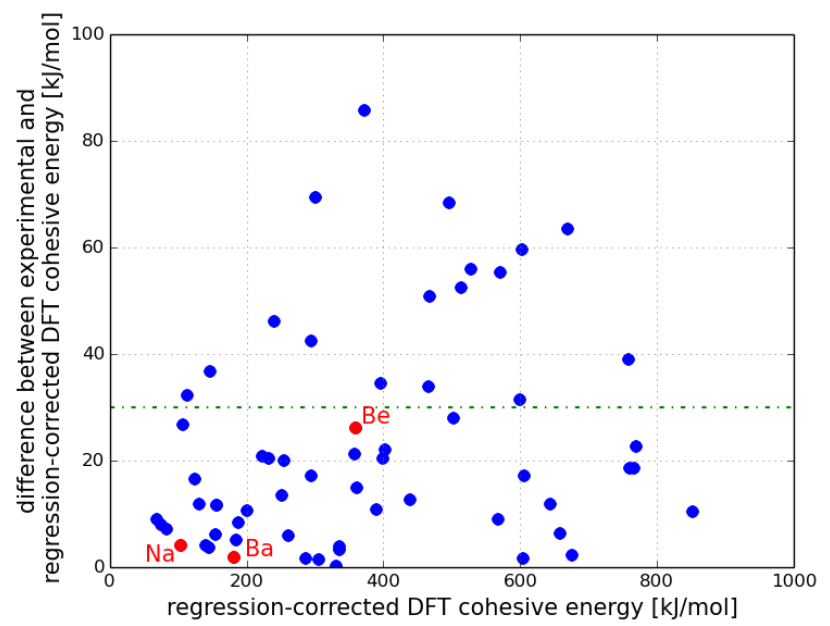

(b)

Figure 1. (Colour online) Absolute values of the difference between DFT predictions and thermally corrected experimental values for (a) the equilibrium volume, plotted as a function of the DFT volume and (b) the cohesive energy, plotted as a function of the DFT cohesive energy. ${ }^{6}$ Three example materials are highlighted in red. All DFT quantities were corrected for systematic deviations from experiment by means of a linear regression, following the procedure described by Lejaeghere et $a l .{ }^{6}$. For the volume no obvious absolute (dotdashed line) or relative behaviour (dashed line) is observed, while the cohesive energy is characterized by an absolute error bar (dot-dashed line). by the PBE functional) have been corrected for, allowing for a better stochastic description of the remaining errors.

\section{ANSATZ}

Before dealing with the equilibrium volume, we first focus on the energy. Indeed, besides electron densities or wave functions, the basic quantities in first-principles methods are cohesive energies or, more precisely, cohesive energy differences. They are used to evaluate the relative stability of two configurations, such as in formation energies. In addition, many other properties are derived from energy differences. Think of elastic moduli, for example, which represent second derivatives of the total energy with respect to deformation. Since the equilibrium volume relates to the absolute minimum of the potential energy surface, this quantity too depends on energy differences.

The primary ansatz of this study is that after correcting for systematic deviations, the error bar on cohesive energy (differences) per atom is absolute, i.e. not proportional to the cohesive energy (difference) itself. Indeed, a relative error is unlikely, as small cohesive energy (differences) may arise from the cancellation of large energetic contributions, thus not necessarily giving rise to smaller errors. The assumption of an absolute error bar is also consistent with observed distributions of cohesive energies (see Figure 1(b)) and formation energies. ${ }^{22,23}$. When assessing a large and diverse set of materials, the errors may then be considered to assume a Gaussian distribution, and only the elimination of systematic deviations makes it possible to treat all materials on equal footing. Note that each type of cohesive energy difference is characterized by its own error bar. The energy difference between two almost identical structures will always be small, and hence the error will be too. This is the case for two slightly different volumes of the same material, for example. We account for this dependency of the error on the volume, as will be discussed later.

If the fundamental error is an error on energy differences, the errors on derived quantities, such as the volume, must depend on it. Whether the error on the volume should be absolute or relative hence follows from the absolute character of the energy error. The easiest way to determine the relation between the volume error and the energy error, is by looking at the inverse problem: rather than starting from a certain energy error and investigating what volume changes yield equations of state (EOS) within that error bar, it is more straightforward to directly evaluate the energy change associated with a change in equilibrium volume. We first treat this problem in an analytic yet approximate way, ascertaining the most important trends. Afterwards, we apply a more rigorous numerical method based on a Monte Carlo procedure. 


\section{ANALYTIC APPROXIMATION}

To analytically assess the relation between errors on the energy and on the EOS parameters (such as the equilibrium volume), we examine how the shape of the EOS influences the energy difference between two fixed volumes, $E\left(\alpha V_{0}^{\prime}\right)-E\left(V_{0}^{\prime}\right) \equiv \Delta E(\alpha)$, with $V_{0}^{\prime}$ a volume close to the equilibrium volume and $\alpha$ close to 1 (see coloured vertical lines near the $E$ axis in Figure 2(a)). We use the Birch-Murnaghan EOS with zero reference energy:

$$
\begin{aligned}
E(V) & =\frac{9 V_{0} B_{0}}{16}\left\{\left[\left(\frac{V_{0}}{V}\right)^{2 / 3}-1\right]^{3} B_{1}\right. \\
& \left.+\left[\left(\frac{V_{0}}{V}\right)^{2 / 3}-1\right]^{2}\left[6-4\left(\frac{V_{0}}{V}\right)^{2 / 3}\right]\right\}
\end{aligned}
$$

where $V_{0}$ represents the equilibrium volume, $B_{0}$ the bulk modulus and $B_{1}$ the pressure derivative of the bulk modulus. To first order, the uncertainty on $\Delta E(\alpha)$ can be written as:

$\delta(\Delta E(\alpha))=\frac{\partial \Delta E(\alpha)}{\partial V_{0}} \delta V_{0}+\frac{\partial \Delta E(\alpha)}{\partial B_{0}} \delta B_{0}+\frac{\partial \Delta E(\alpha)}{\partial B_{1}} \delta B_{1}$

To get a first crude estimate of the sensitivity of the equation-of-state parameters to errors on the energy, we will consider one error on an EOS parameter at a time while the other ones are omitted from the error propagation. In that case, the following expressions are obtained:

$$
\begin{aligned}
\delta V_{0} & \approx\left(\frac{\partial \Delta E(\alpha)}{\partial V_{0}}\right)^{-1} \delta(\Delta E(\alpha)) \\
\delta B_{0} & \approx\left(\frac{\partial \Delta E(\alpha)}{\partial B_{0}}\right)^{-1} \delta(\Delta E(\alpha)) \\
\delta B_{1} & \approx\left(\frac{\partial \Delta E(\alpha)}{\partial B_{1}}\right)^{-1} \delta(\Delta E(\alpha))
\end{aligned}
$$

Evaluation at $V_{0}^{\prime}=V_{0}$ then leads to:

$$
\begin{aligned}
\delta V_{0} & \approx \frac{16}{9 B_{0}\left[f_{1}(\alpha) B_{1}+f_{2}(\alpha)\right]} \delta(\Delta E(\alpha)) \\
\delta B_{0} & \approx \frac{16}{9 V_{0}\left[g_{1}(\alpha) B_{1}+g_{2}(\alpha)\right]} \delta(\Delta E(\alpha)) \\
\delta B_{1} & \approx \frac{16}{9 B_{0} V_{0} g_{1}(\alpha)} \delta(\Delta E(\alpha))
\end{aligned}
$$

with

$$
\begin{gathered}
f_{1}(\alpha)=\left[3\left(\frac{1}{\alpha}\right)^{2 / 3}-1\right]\left[\left(\frac{1}{\alpha}\right)^{2 / 3}-1\right]^{2} \\
f_{2}(\alpha)=\left[\left(\frac{1}{\alpha}\right)^{2 / 3}-1\right]\left[-12\left(\frac{1}{\alpha}\right)^{4 / 3}\right. \\
\left.+\frac{62}{3}\left(\frac{1}{\alpha}\right)^{2 / 3}-6\right]
\end{gathered}
$$

and

$$
\begin{gathered}
g_{1}(\alpha)=\left[\left(\frac{1}{\alpha}\right)^{2 / 3}-1\right]^{3} \\
g_{2}(\alpha)=\left[\left(\frac{1}{\alpha}\right)^{2 / 3}-1\right]^{2}\left[6-4\left(\frac{1}{\alpha}\right)^{2 / 3}\right]
\end{gathered}
$$

Although none of the $\delta X$ is found to be proportional to $X$, we need to take into account that in reality, it is impossible to vary only one EOS parameter at a time. On the contrary, for real materials $V_{0}$ and $B_{0}$ are correlated: a material with a large equilibrium volume usually has a much smaller bulk modulus, and vice versa (Fig. 3). When assuming an inverse proportionality, we see that the errors on the volume and the bulk modulus become approximately relative. The error on $B_{1}$ remains absolute, on the other hand, as $B_{1}$ is largely unrelated to $V_{0}$ and $B_{0}$ (see the Supplementary Material).

\section{MONTE CARLO APPROACH}

The preceding analytic approach gives a first impression of how the errors on the EOS parameters are interdependent. Do note, however, that none of these conclusions takes into account the occurrence of mixed changes, such as $\delta V_{0} \delta B_{0}$, or higher-order terms in $\delta V_{0}$. The exact behaviour can be validated using a Monte Carlo (MC) approach (see Figure 2(b)). In that case, all parameters of a reference EOS are varied simultaneously (yet independently) and in a random fashion according to a Metropolis algorithm. ${ }^{24,25}$ By only accepting changes that are consistent with an error model for the energy differences, the observed spread (standard deviation) on the EOS parameters can be interpreted as an error measure. Note that this procedure improves on the efficiency and statistical rigour of the method previously applied by some of the present authors, ${ }^{26}$ where a slightly different approach was used to derive uncertainties on EOS parameters from the $E(V)$ fitting error.

In this study, we investigate the effects within the $0.94 V_{0}$ to $1.06 V_{0}$ interval, as this is also the volume range in which $E(V)$ or $P(V)$ data from DFT are typically fitted. ${ }^{6,11-13,15}$ Because we are now dealing with an entire volume range - the previous analytic treatment only considered the energy difference between two fixed volumes - it is necessary to properly take into account the volume dependence of the energy error. As mentioned earlier, we expect the error for a particular material to increase as the individual energy terms in the many-body Hamiltonian become larger in magnitude. For the cohesive energy, this implies that errors will grow as the volume decreases, since the interaction between the different atoms is then stronger. As a first approximation, we therefore assume the energy error to directly scale with the particle 


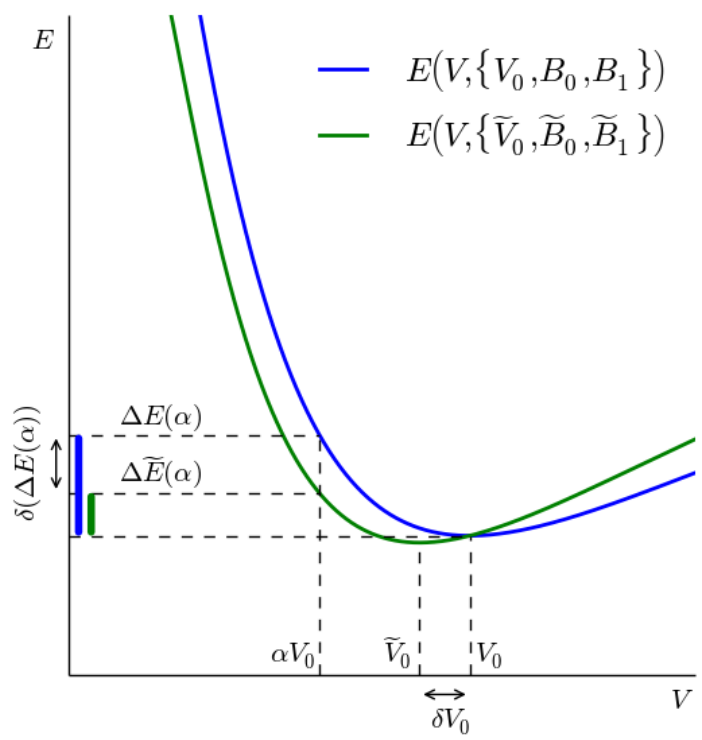

(a)
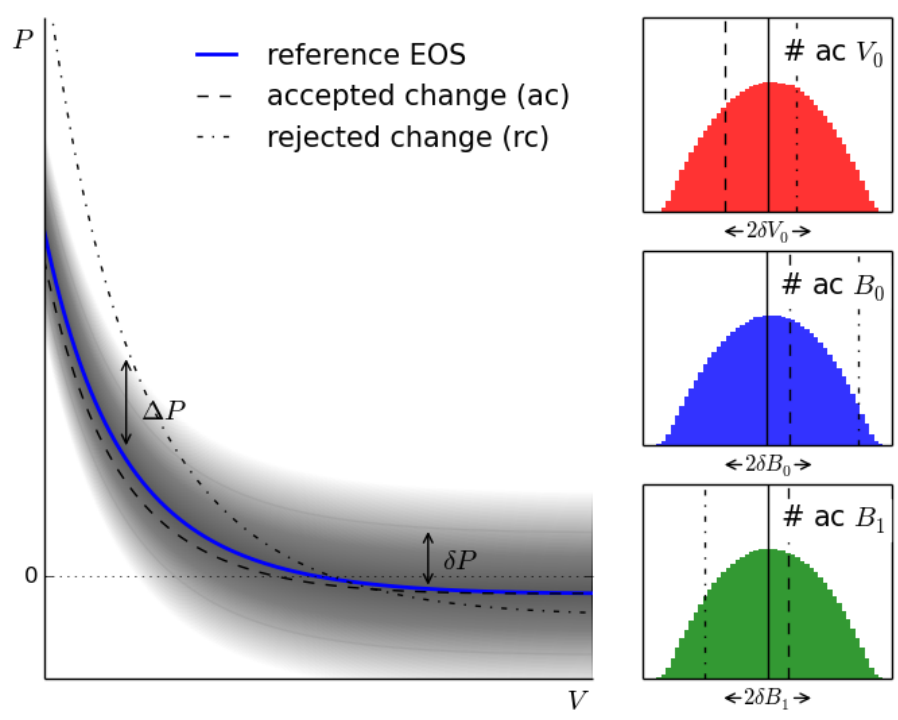

(b)

Figure 2. (Colour online) (a) Analytic and (b) Monte Carlo determination of the error bar on equation-of-state parameters. (a) Shifting the parameters of an EOS by $\left(\delta V_{0}, \delta B_{0}, \delta B_{1}\right)$, i.e. from $\left(V_{0}, B_{0}, B_{1}\right)$ to $\left(\tilde{V}_{0}, \tilde{B}_{0}, \tilde{B}_{1}\right)$, causes the energy difference between $V_{0}$ and $\alpha V_{0}$ to change by $\delta(\Delta E(\alpha)$ ) (see Equation (2)). (b) By randomly shifting the parameters of an EOS and only accepting changes in accordance with the error distribution of the pressure (grey area), we can store the parameters of the accepted EOS curves and assess their distributions (number of accepted EOS parameters per bin, \# ac, in the histograms on the right). The standard deviations of these distributions then allow the definition of corresponding error bars $\left(\delta V_{0}, \delta B_{0}\right.$ and $\delta B_{1}$ ). The figure shows how two proposed changes are accepted (dashed line) or rejected (dot-dashed line) depending on how the pressure change $\Delta P$ relates to the pressure error $\delta P$. The parameters of the accepted and the rejected EOS are indicated with the same line style in the histograms.

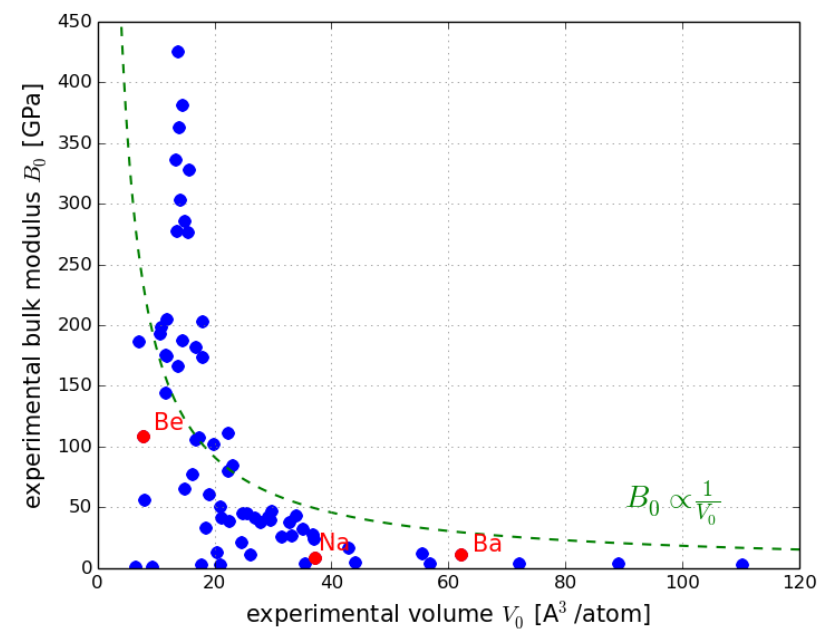

Figure 3. (Colour online) Experimental bulk modulus in GPa versus the experimental equilibrium volume in $\AA^{3}$ /atom, displayed for the pure elemental crystals. ${ }^{6}$ Three example materials are highlighted in red. An approximately inversely proportional relation is observed. interaction, following the common $1 / r$ behaviour:

$$
\delta E_{c o h}(V)=\epsilon\left(\frac{V_{0}}{V}\right)^{1 / 3}
$$

Note that we now use the notation $\delta X$ for the error on property $X$ in a more general sense: rather than indicating one particular change in $X, \delta X$ now denotes an overall measure of the error distribution. Equation (13) causes the error on the cohesive energy to become zero for $V \rightarrow \infty$ (since the cohesive energy is then zero by definition), while for $V=V_{0}$, an error bar of $\delta E_{c o h}\left(V_{0}\right)=\epsilon$ is found. This allows us to tune the error bar to previously established cohesive energy errors. ${ }^{6}$ We will later show that the exact shape of the error function does not much affect the MC error estimates.

Equation (13) corresponds to the expected error on energy differences with the crystal at infinite volume. However, in our Monte Carlo approach, we only consider volumes in a limited interval, and do not possess information on the energy of infinitely separated atoms. We hence need to translate the equation of state into an energy difference inherent to the studied volume range, and determine the corresponding error. Rather than choosing an arbitrary reference state in that volume range, to which the EOS energies can be compared, we choose to transform the energy-based EOS to a pressure-based 
one. Indeed, each pressure intrinsically corresponds to an energy difference, without the need for one particular reference energy:

$$
P(V)=-\frac{\partial E}{\partial V}
$$

The energy error then translates into:

$$
\delta P(V)=-\frac{\partial}{\partial V} \delta E_{c o h}(V)=\frac{\epsilon}{3 V_{0}}\left(\frac{V_{0}}{V}\right)^{4 / 3}
$$

In our MC simulation, we therefore accept changes in the EOS parameters based on how the resulting pressure change $\Delta P$ relates to $\delta P$ (see Figure 2(b)).

To perform the Monte Carlo calculations in a statistically sound way, we express this acceptance criterion in terms of a continuous distribution function rather than a rigorous cutoff. Assuming a normal distribution of the energy errors, the squared sum of normalized pressure changes at three different volumes should follow a $\chi_{3}^{2}$ distribution. We therefore use this to define the acceptance criterion for each step in the Markov chain. More details on the acceptance criterion, as well as on each of the individual Monte Carlo calculations, can be found in the Supplementary Material.

We now apply this approach to a Birch-Murnaghan equation of state. In that case, the pressure is given by:

$$
\begin{aligned}
P(V)=\frac{3 B_{0}}{2} & {\left[\left(\frac{V_{0}}{V}\right)^{7 / 3}-\left(\frac{V_{0}}{V}\right)^{5 / 3}\right] } \\
\times & \left\{1+\frac{3}{4}\left(B_{1}-4\right)\left[\left(\frac{V_{0}}{V}\right)^{2 / 3}-1\right]\right\}
\end{aligned}
$$

We impose an energy error $\epsilon$ of $0.15 \mathrm{eV} /$ atom, which is of the order of magnitude of the expected difference between DFT-PBE and experiment. ${ }^{6}$ In this way, we obtain the error bars for $V_{0}, B_{0}$ and $B_{1}$ depicted in Figure 4 for 64 different initial EOS parameter sets. This reveals a number of interesting trends. $\delta V_{0}$ is largely independent of the volume and the bulk modulus derivative, but scales inversely with the bulk modulus. $\delta B_{0}$ is largely independent of the bulk modulus and the bulk modulus derivative, but scales inversely with the equilibrium volume. $\delta B_{1}$ is largely independent of the bulk modulus derivative, but scales inversely with the product of the bulk modulus and the equilibrium volume. Each of these conclusions was already derived analytically in Equations (6), (7) and (8) for independent variations of $V_{0}, B_{0}$ and $B_{1}$, and is now confirmed for the general case.

We can also take a closer look at the behaviour of the error bars. Figure 5 displays a number of $1 \mathrm{D}$ cross sections for the 3D data sets presented in Figure 4. We readily recognize the above mentioned trends again, but more subtle dependencies appear as well. $\delta V_{0}$, for example, is not only inversely proportional to $B_{0}$, but it increases very slightly as $V_{0}$ rises. In the same way, $\delta B_{0}$ not only drops steadily with volume, but also decreases slowly as a function of $B_{0}$. Both trends are found to follow from a shift in the average EOS parameters during the course of the Monte Carlo simulation. Indeed, due to the skewed distribution of $V_{0}$ and $B_{0}$, their averages do not coincide with the original reference values. Instead, starting from a larger $B_{0}$ leads to distributions with a larger average $V_{0}$, and a large initial $V_{0}$ gives rise to a smaller average $B_{0}$ (see Figure 6). The observed dependencies are therefore in line with the trends mentioned earlier.

Another noticeable trend is the slight increase of $\delta B_{0}$ for larger $B_{1}$ values. Contrary to the dependencies of $\delta V_{0}$ and $\delta B_{0}$ on $V_{0}$ and $B_{0}$, respectively, changes in the mean values are not responsible for this phenomenon. Because this behaviour is also not found analytically, it must be due to correlations between changes in the three EOS parameters.

Finally, the dependencies of $\delta V_{0}$ and $\delta B_{1}$ on $B_{1}$ are not significant: repeating the same MC simulation several times yields fluctuations that are at least as large. We can therefore conclude that the errors are mostly decoupled, and that the analytically derived behaviour reproduces the most important trends.

We could wonder whether the above conclusions are also valid for another volume dependence of the energy error, or for other equations of state. We therefore redo the analysis for a Birch-Murnaghan equation of state with constant pressure error $\delta P(V)=\delta P$ and for a Vinet equation of state:

$$
\begin{aligned}
P(V)=3 & B_{0}\left(\frac{V}{V_{0}}\right)^{-2 / 3}\left[1-\left(\frac{V}{V_{0}}\right)^{1 / 3}\right] \\
\times & \exp \left\{\frac{3}{2}\left(B_{1}-1\right)\left[1-\left(\frac{V}{V_{0}}\right)^{1 / 3}\right]\right\}
\end{aligned}
$$

with the error in Eq. (15). Full data are available in the Supplementary Material.

When using a constant pressure error, we find that the resulting error bars are almost identical to the original ones: less than $3 \%$ difference between a constant pressure error and a volume-dependent one. This indicates that the exact volume dependence of the error bar has only a limited influence on our results. Substantial deviations are only possible when both the volume and the bulk modulus are small (up to $16 \%$ in $\delta B_{1}$ ). In that case, a shallow equation of state with a large pressure error bar is obtained (see Eq. (15)), so the effect of the volume dependence of the error bar becomes more pronounced.

Most observed error bars are largely the same when using a Vinet EOS: $76 \%$ of them vary by $5 \%$ or less from the Birch-Murnaghan results. However, the remaining error bars are significantly larger. This is because a Vinet $P(V)$ curve is inherently less sensitive to its EOS parameters. Indeed, its exponential form (17) ensures a smooth transition between the zero pressure at the equilibrium volume and the asymptotic one at infinite volume. As a consequence, Vinet curves with quite large changes in $V_{0}$ 

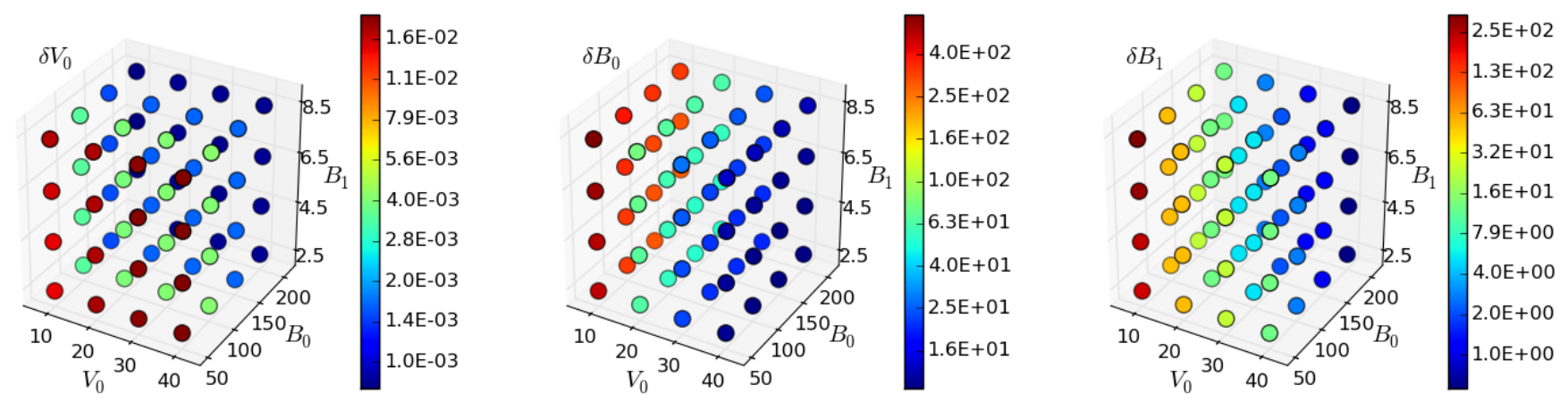

Figure 4. (Colour online) Error bars $\delta V_{0}$ (in $\AA^{3} /$ atom), $\delta B_{0}$ (in GPa) and $\delta B_{1}$ (dimensionless) as a function of the equilibrium volume, bulk modulus and bulk modulus derivative of a Birch-Murnaghan EOS. All results were obtained from $2 \cdot 10^{7}$-step MC simulations.

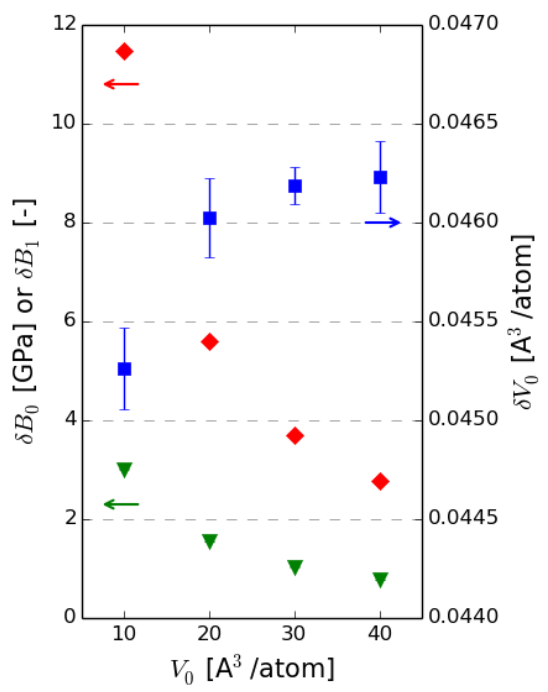

(a)

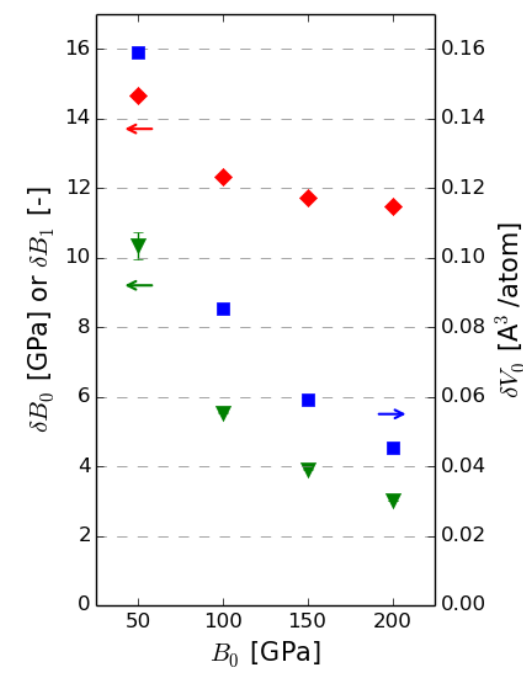

(b)

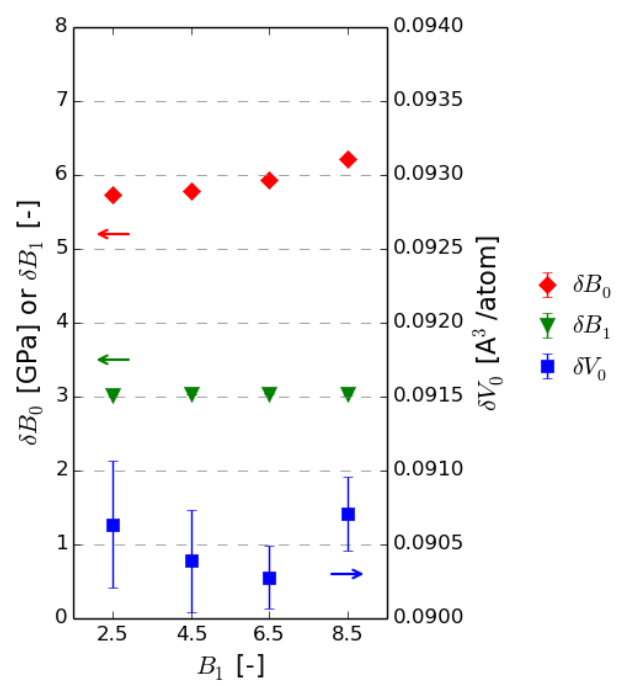

(c)

Figure 5. (Colour online) Error bars for the equilibrium volume (right $y$ axis), bulk modulus and bulk modulus derivative (left $y$ axis) of a Birch-Murnaghan EOS (a) as a function of the volume and with $B_{0}=200 \mathrm{GPa}, B_{1}=2.5$, (b) as a function of the bulk modulus and with $V_{0}=10 \AA^{3}$ /atom, $B_{1}=2.5$, and (c) as a function of the bulk modulus derivative and with $V_{0}=20 \AA^{3} /$ atom, $B_{0}=100 \mathrm{GPa}$. All results were obtained from $2 \cdot 10^{7}$-step MC simulations. The error bar symbols depict the standard deviation over 4 equivalent MC runs.

or $B_{1}$ can still yield reasonable pressures, especially when the pressure error bar was large to begin with (i.e., when $V_{0}$ is small) or the EOS shallow (i.e., when $B_{0}$ is small). The Vinet error bars may be $20 \%$ up to even $60 \%$ larger than the Birch-Murnaghan ones in those cases. Nevertheless, except maybe for $\delta V_{0}$ as a function $V_{0}$, which suffers from huge error bars, the trends observed in Figure 5 are retained.

Finally, we also check how the magnitude of the energy error $\epsilon$ influences our results. We therefore analyse a single reference Birch-Murnaghan EOS - with $V_{0}=20 \AA^{3} /$ atom, $B_{0}=50 \mathrm{GPa}$ and $B_{1}=4.5-$ and vary $\epsilon$ in Eq. (15). The resulting $\delta V_{0}, \delta B_{0}$ and $\delta B_{1}$ are shown in Figure 7 . We find the errors on $V_{0}, B_{0}$ and
$B_{1}$ to scale linearly with $\epsilon$, validating the main approximation in our analytic approach. Because of the simplicity of this relation, the results of the present study may even straightforwardly be extrapolated to different benchmark situations: only the energy error $\epsilon$ on the method of interest is required to rescale the error bars on the EOS parameters reported here. An explicit determination of $V_{0}, B_{0}$ and $B_{1}$ is not necessary. Such an error bar rescaling approach is not only valid to assess the difference between predictions of a particular level of theory and experimental values, but for example also to compare the predictions of different codes with respect to the same level of theory. ${ }^{6,27}$ 


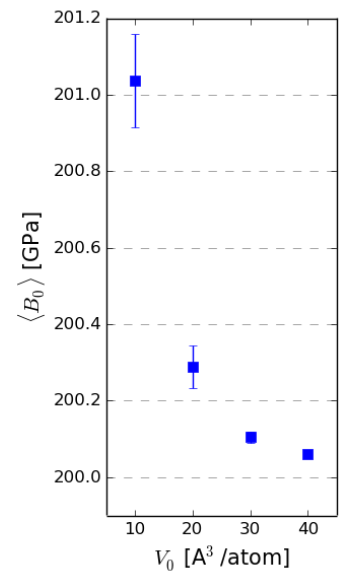

(a)

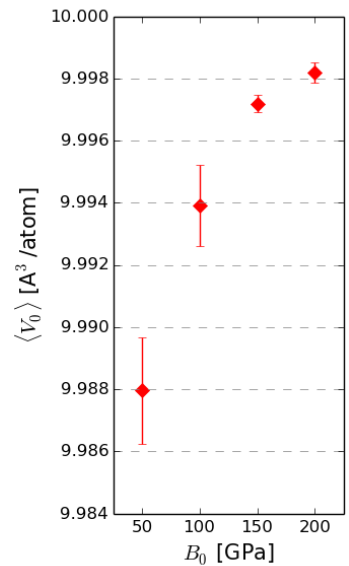

(b)
Figure 6. (Colour online) (a) Average value of $B_{0}$ as a function of the volume, with $B_{0}=200 \mathrm{GPa}, B_{1}=2.5$, and (b) average value of $V_{0}$ as a function of the bulk modulus, with $V_{0}=10 \AA^{3} /$ atom, $B_{1}=2.5$. All results were obtained from $2 \cdot 10^{7}$-step MC simulations. The error bar symbols depict the standard deviation over 4 equivalent $\mathrm{MC}$ runs.

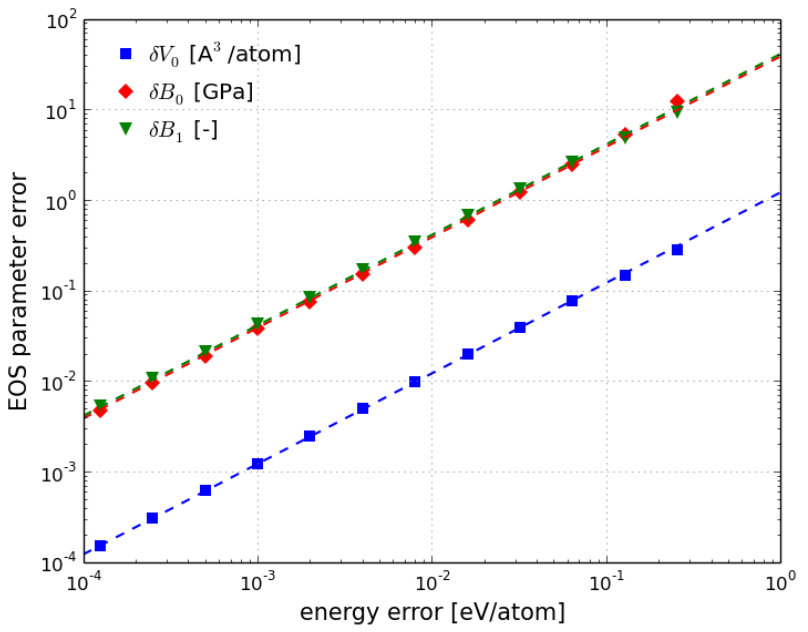

Figure 7. (Colour online) Error bars $\delta V_{0}$ (in $\AA^{3} /$ atom), $\delta B_{0}$ (in GPa) and $\delta B_{1}$ (dimensionless) as a function of $\epsilon$ for a Birch-Murnaghan EOS with $V_{0}=20 \AA^{3} /$ atom, $B_{0}=50 \mathrm{GPa}$ and $B_{1}=4.5$. All results were obtained from $2 \cdot 10^{7}$-step MC simulations.

\section{CONCLUSION}

In conclusion, we have now elucidated the behaviour of equation-of-state errors in first-principles predictions. These errors are present because of inherent approximations, such as the use of approximate exchangecorrelation functionals in DFT, leading to a less-thanperfect agreement with experimental values. Systematic deviations can be corrected for, but the character of the remaining errors has remained elusive until now. The mismatch between the theoretical and experimental volume of a crystal, for example, is described in literature in terms of both absolute and relative errors, with a preference for the latter. We investigated this problem by means of both an analytic treatment and a more accurate Monte Carlo approach. By retracing the errors on the equation of state to errors on energy differences, we were able to demonstrate that the error on the equilibrium volume is actually neither absolute nor relative (see Figure 1(a)), but inversely proportional to the bulk modulus (see Figure 8). Because large bulk moduli often go hand in hand with small volumes, the error appears to behave as a relative error over a large volume range. It is therefore justified to report percentage errors on equilibrium volumes - approximately $3 \%$ on top of a $4 \%$ systematic overestimation for PBE - although better error estimates are derived from the inverse bulk modulus - about $35 / B_{0}$ for PBE (with $\delta V_{0}$ in $\AA^{3} /$ atom and $B_{0}$ in GPa). When selecting one functional over another, or when designing new functionals, such aspects need to be taken into account. Indeed, Civalleri et al. showed that the used method of error quantification may influence the outcome. ${ }^{17}$ Moreover, reporting the computational error bars may be considered a good practice for any first-principles study. The PBE error bars reported here are generally applicable, and similar measures can be determined for other levels of theory using appropriate benchmark results for $V_{0}, B_{0}$ and $B_{1}$, or by rescaling based on the error on the energy (see Figure 7).

In this work, we did not take into account experimental error bars, which may certainly provide valuable insights for materials properties that are harder to measure than the volume. We did show this energy-based error assessment also to be applicable to other EOS properties, in particular the bulk modulus (with an approximately relative error bar) and the bulk modulus derivative (with an absolute error bar). This opens new perspectives for the large-scale use of first-principles methods, since such an approach allows us to recognize which materials and materials properties are most prone to deviations from experiment, and which predictions are safe to use.

\section{ACKNOWLEDGEMENTS}

This work benefited from financial support from the Research Board of Ghent University (BOF), the Research Foundation - Flanders (FWO) (project number G0E0116N) and BELSPO in the frame of IAP-PAI $\mathrm{P} 7 / 05$. Funding was also received from the European Research Council under the European Community's Seventh Framework Programme [FP7(2007-2013) ERC grant agreement number 240483] and from the European Union Horizon 2020 research and innovation programme [consolidation ERC grant, agreement No. 647755-DYNPOR (2015-2020)]. S.C. acknowledges financial support from OCAS NV by an OCAS-endowed chair at Ghent Univer- 


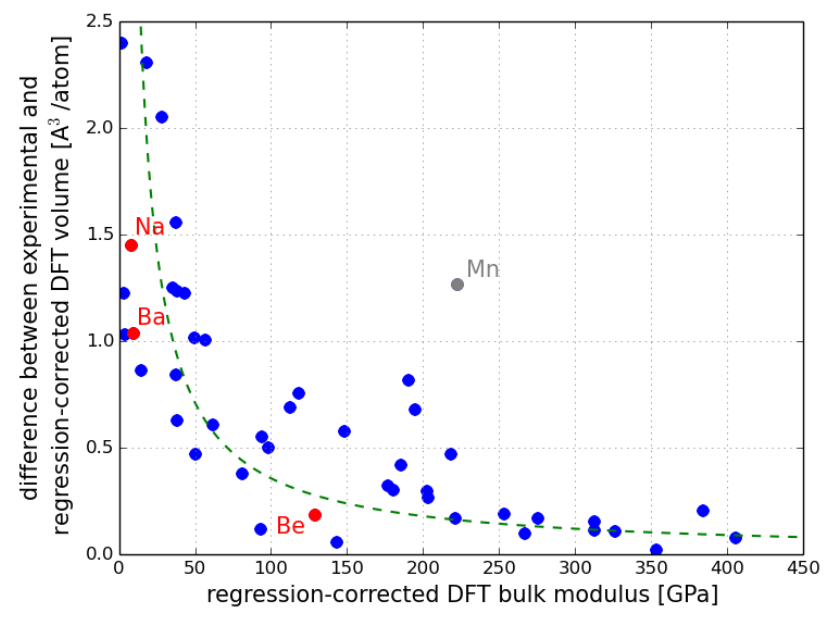

Figure 8. (Colour online) Absolute values of the difference between DFT predictions and thermally corrected experimental values for the equilibrium volume, plotted as a function of the DFT bulk modulus. ${ }^{6}$ Three example materials are highlighted in red. All DFT quantities were corrected for systematic deviations from experiment by means of a linear regression, following the procedure described by Lejaeghere et al. ${ }^{6} \mathrm{~A}$ good correspondence to an inverse behaviour is observed (dashed line), in accordance with Eq. (6) and Figure 5(b). Mn (223, 1.3 ) (in grey) stands out as an outlier, but its bulk modulus is severely overestimated and its volume underestimated, contrary to the PBE trends. sity. The computational resources and services used in this work were provided by Ghent University (Stevin), the Hercules Foundation (Tier-1 Flemish Supercomputer Infrastructure), and the Flemish Government - Department of EWI.
1 A.K. Rappé, C.J. Casewit, K.S. Colwell, W.A. Goddard, and W.M. Skiff, "UFF, a full periodic table force field for molecular mechanics and molecular dynamics simulations," J. Am. Chem. Soc. 114, 10024-10035 (1992).

2 Louis Vanduyfhuys, Steven Vandenbrande, Toon Verstraelen, Rochus Schmid, Michel Waroquier, and Veronique Van Speybroeck, "QuickFF: A program for a quick and easy derivation of force fields for metal-organic frameworks from ab initio input," J. Comput. Chem. 36, 1015-1027 (2015).

3 George H. Booth, Andreas Grüneis, Georg Kresse, and Ali Alavi, "Towards an exact description of electronic wavefunctions in real solids," Nature 493, 365-370 (2013).

${ }^{4}$ P. Hohenberg and W. Kohn, "Inhomogeneous electron gas," Phys. Rev. 136, B864 (1964).

${ }^{5}$ W. Kohn and L. J. Sham, "Self-consistent equations including exchange and correlation effects," Phys. Rev. 140, A1133 (1965).

${ }^{6}$ K. Lejaeghere, V. Van Speybroeck, G. Van Oost, and S. Cottenier, "Error estimates for solid-state densityfunctional theory predictions: An overview by means of the ground-state elemental crystals," Crit. Rev. Solid State Mater. Sci. 39, 1-24 (2014).

7 Larry A. Curtiss, Krishnan Raghavachari, Paul C. Redfern, and John A. Pople, "Assessment of Gaussian-2 and density functional theories for the computation of enthalpies of formation," J. Chem. Phys. 106, 1063-1079 (1997).
8 Y. Zhao and D. G. Truhlar, "Density functionals with broad applicability in chemistry," Acc. Chem. Res. 41, 157-167 (2008).

9 Martin Korth and Stefan Grimme, "“Mindless" DFT benchmarking," J. Chem. Theory Comput. 5, 993-1003 (2009).

10 Lars Goerigk and Stefan Grimme, "A thorough benchmark of density functional methods for general main group thermochemistry, kinetics, and noncovalent interactions," Phys. Chem. Chem. Phys. 13, 6670-6688 (2011).

11 Stefan Kurth, John P. Perdew, and Peter Blaha, "Molecular and solid-state tests of density functional approximations: LSD, GGAs, and meta-GGAs," Int. J. Quantum Chem. 75, 889-909 (1999).

12 Viktor N. Staroverov, Gustavo E. Scuseria, Jianmin Tao, and John P. Perdew, "Tests of a ladder of density functionals for bulk solids and surfaces," Phys. Rev. B 69, 075102 (2004).

13 Viktor N. Staroverov, Gustavo E. Scuseria, Jianmin Tao, and John P. Perdew, "Erratum: Tests of a ladder of density functionals for bulk solids and surfaces [Phys. Rev. B 69, 075102 (2004)]," Phys. Rev. B 78, 239907(E) (2008).

14 Fabien Tran, Robert Laskowski, Peter Blaha, and Karlheinz Schwarz, "Performance on molecules, surfaces, and solids of the Wu-Cohen GGA exchange-correlation energy functional," Phys. Rev. B 75, 115131 (2007).

15 Gábor I. Csonka, John P. Perdew, Adrienn Ruzsinszky, Pier H. T. Philipsen, Sébastien Lebègue, Joachim Paier, 
Oleg A. Vydrov, and János G. Ángyán, "Assessing the performance of recent density functionals for bulk solids," Phys. Rev. B 79, 155107 (2009).

16 Laurids Schimka, René Gaudoin, Jiří Klimeš, Martijn Marsman, and Georg Kresse, "Lattice constants and cohesive energies of alkali, alkaline-earth, and transition metals: Random phase approximation and density functional theory results," Phys. Rev. B 87, 214102 (2013).

17 Bartolomeo Civalleri, Davide Presti, Roberto Dovesi, and Andreas Savin, "On choosing the best density functional approximation," Chem. Modell. 9, 168-185 (2012).

18 Kurt Lejaeghere, Jan Jaeken, Veronique Van Speybroeck, and Stefaan Cottenier, "Ab initio based thermal property predictions at a low cost: An error analysis," Phys. Rev. B 89, 014304 (2014).

19 Pascal Pernot, Bartolomeo Civalleri, Davide Presti, and Andreas Savin, "Prediction uncertainty of density functional approximations for properties of crystals with cubic symmetry," J. Phys. Chem. A 119, 5288-5304 (2015).

${ }^{20}$ Karl K. Irikura, Russell D. III Johnson, and Raghu N. Kacker, "Uncertainty associated with virtual measurements from computational quantum chemistry models," Metrologia 41, 369-375 (2004).

21 J. J. Mortensen, K. Kaasbjerg, S. L. Frederiksen, J. K. Nørskov, J. P. Sethna, and K. W. Jacobsen, "Bayesian error estimation in density-functional theory," Phys. Rev. Lett. 95, 216401 (2005).
22 Geoffroy Hautier, Shyue Ping Ong, Anubhav Jain, Charles J. Moore, and Gerbrand Ceder, "Accuracy of density functional theory in predicting formation energies of ternary oxides from binary oxides and its implication on phase stability," Phys. Rev. B 85, 155208 (2012).

23 James E. Saal, Scott Kirklin, Muratahan Aykol, Bryce Meredig, and C. Wolverton, "Materials design and discovery with high-throughput density functional theory: the Open Quantum Materials Database (OQMD)," JOM 65, 1501-1509 (2013).

24 Nicholas Metropolis, Arianna W. Rosenbluth, Marshall N. Rosenbluth, Augusta H. Teller, and Edward Teller, "Equation of state calculations by fast computing machines," J. Chem. Phys. 21, 1087-1092 (1953).

${ }^{25}$ W. K. Hastings, "Monte Carlo sampling methods using Markov chains and their applications," Biometrika 57, 97109 (1970).

26 Danny E. P. Vanpoucke, Kurt Lejaeghere, Veronique Van Speybroeck, Michel Waroquier, and An Ghysels, "Mechanical properties from periodic plane wave quantum mechanical codes: The challenge of the flexible nanoporous MIL-47(V) framework," J. Phys. Chem. C 119, 2375223766 (2015)

27 K. Lejaeghere, G. Bihlmayer, T. Björkman, P. Blaha, S. Blügel, V. Blum, D. Caliste, I.E. Castelli, S.J. Clark, A. Dal Corso et al., "The Kohn-Sham equation of state for elemental solids: a solved problem," (2015), submitted. 